\title{
Cálculo da rotação, do torque e da potência de motores elétricos para transmissão por parafusos de potência
}

\author{
Luciano Galdino' \\ Recebido em: 10/11/2014. Aprovado em: 04/12/2014. Disponibilizado em: 26/12/2014
}

1. Luciano Galdino é Mestre em Ciências Exatas e da Terra na área de Física Nuclear pela USP, especializado em Física pela USP e Licenciado em Matemática pela UNG. Professor de Física e Elementos de Máquina na Faculdade ENIAC. E-mail: lucianogaldino1@yahoo.com.br.

\section{Resumo}

Este artigo apresenta detalhadamente os cálculos necessários para a obtenção do torque, da potência e da rotação de motores elétricos que atuam com o objetivo de transformar um movimento angular (rotação) em movimento linear (translação retilínea), utilizando o parafuso de potência (também chamado de parafuso de avanço ou simplesmente fuso) como elemento de transmissão, tanto para o movimento vertical como para o movimento horizontal. Para isso foi elaborada uma breve descrição sobre as principais características de motores elétricos e a sua conversão eletromecânica, assim como uma descrição dos parafusos de potência, destacando o tipo de rosca adequado para determinadas transmissões e a eficiência desse tipo de transmissão. São apresentados resultados de estudos sobre coeficientes de atrito para a combinação de alguns materiais do parafuso de potência e de sua porca e também curvas de eficiência em função do ângulo de avanço e do coeficiente de atrito.

Palavras-chave: Torque. Potência. Rotação. Motores. Parafuso.

\begin{abstract}
This article presents in detail the calculations required to obtain the torque, power and speed of electric motors that operate with the goal of transforming an angular motion (rotation) into linear motion (rectilinear translation), using the power screw (also called a lead screw or simply screw) as the driving element for both the vertical motion and for horizontal movement. For this we created a brief description of the main characteristics of electric motors and their electromechanical conversion, as well as a description of power screws, highlighting the type of thread suitable for certain transmissions and the efficiency of this type of transmission. Results of studies on the coefficients of friction for a combination of some materials power screw and nut and also its efficiency curves depending on the lead angle and friction coefficient are presented.
\end{abstract}

Keywords: Torque. Power. Rotation. Engines. Screw. 


\section{Introdução}

O projetista, durante a elaboração de um projeto, deve inicialmente fazer um levantamento das grandezas que serão determinadas de acordo com a finalidade do projeto e elaborar uma forma de calcular as grandezas desconhecidas, sempre buscando a simplificação e o barateamento do projeto. $\mathrm{Na}$ literatura não é encontrada uma forma clara e detalhada de como estimar as características mecânicas principais (rotação, torque e potência) que um motor deverá desenvolver para o projeto de um mecanismo que utilize um parafuso de potência como elemento de transmissão e com o objetivo de transformar o movimento angular em movimento linear.

Os motores elétricos são dispositivos muito empregados nas indústrias não apenas para aplicações de movimentos angulares (rotação), mas também em aplicações que utilizem transmissões de movimento. Existem diversos tipos de motores, alguns que trabalham com corrente contínua e outros com corrente alternada. $\mathrm{O}$ dimensionamento apresentado neste artigo é generalizado com relação aos motores, não importando o tipo de motor que será utilizado, isto é, os cálculos servem para qualquer tipo de motor, pois a forma de determinar essas grandezas está relacionada com o trabalho mecânico que o motor deverá realizar.

Os parafusos de potência possuem uma infinidade de aplicações na indústria que necessitem de uma transformação de um movimento angular para um movimento linear e com precisão de posicionamento, por esse motivo são muito utilizados para movimentação dos eixos de diversas máquinas operatrizes. Neste trabalho são apresentadas as suas características principais, mas a abordagem principal está relacionada com as características envolvidas na transmissão do movimento, isto é, não será abordado o dimensionamento do parafuso de potência, mas sim as suas características como elemento de transmissão que se enquadram para o dimensionamento de motores, como tipo de rosca, o passo, o número de entradas e a eficiência.

São abordados os estudos das forças envolvidas nesse tipo de transmissão, tanto para o movimento horizontal como para o movimento vertical ascendente e descendente, demonstrando as equações necessárias para tal dimensionamento através de deduções matemáticas detalhadas e sempre levando em consideração o efeito da aceleração para o motor sair do repouso e atingir a sua rotação máxima, pois esse é o momento em que mais se exige do motor e que é desprezado nos livros de elementos de máquinas e de projetos mecânicos. Ao final do trabalho é apresentado também como se calcula a eficiência da transmissão por parafuso de potência.

\section{Motores Elétricos e a Conversão Eletromecânica de Energia}

Um motor elétrico é um dispositivo capaz de transformar energia elétrica em energia mecânica, utilizando o princípio da conversão eletromecânica de energia, sendo que essa energia mecânica é utilizada para acionar diversos tipos de máquinas e equipamentos com grande atuação na indústria (VAZ, 2010).

A conversão eletromecânica de energia envolve a troca de energia entre um sistema elétrico e um sistema mecânico, através de um campo magnético de acoplamento. O processo é essencialmente reversível, exceto por uma pequena quantidade de energia que se perde em aquecimento. Quando a conversão é da forma elétrica para a mecânica, o dispositivo é chamado de motor. (DEL TORO, 2009, p.107). 
Segundo Halliday (2003) corrente alternada (ca) é definida como uma corrente elétrica oscilatória, isto é, uma corrente cujo sentido de propagação é invertido periodicamente. Já a corrente contínua (cc) é definida como uma corrente elétrica nãooscilatória, isto é, uma corrente cujo sentido de propagação é constante no decorrer do tempo.

Quando um sistema elétrico é energizado com corrente alternada têm-se os motores de corrente alternada ou simplesmente motores ca, agora quando um sistema é energizado com corrente contínua têm-se os motores de corrente contínua ou simplesmente motores cc. Tanto para motores ca como para motores cc o torque é governado pelas mesmas leis fundamentais, pois para os dois tipos de motores a definição do torque parte da lei de Ampère. O mesmo é válido para a fem, pois tanto para motores ca como para motores cc, a definição da fem parte da lei de Faraday, isto é, os motores ca não são fundamentalmente diferentes dos motores cc, eles diferem somente em detalhes construtivos, os conceitos básicos são os mesmos (DEL TORO, 2009).
Segundo Del Toro (2009) a conversão eletromecânica de energia está relacionada com a troca de energia elétrica e mecânica, sendo que as grandezas fundamentais no sistema elétrico são a tensão (fem induzida "e") e a corrente (i) e as grandezas fundamentais análogas mecânicas são $\mathrm{O}$ torque $(\mathrm{T})$ e a velocidade angular ( $\omega)$. Como a potência elétrica é o produto da tensão pela corrente (NILSSON, 2009) e a potência mecânica o produto do torque pela velocidade angular (MELCONIAN, 2008), então, pela analogia entre as grandezas, a potência elétrica desenvolvida (potência de saída ou útil) é igual à potência mecânica desenvolvida.

\section{Transmissão por Parafuso de Potência}

As roscas são um conjunto de filetes que contornam corpos cilíndricos, já os parafusos são corpos constituídos por roscas que servem para fixação e transmissão de movimentos (GORDO; FERREIRA, 2012). Os perfis das roscas são diversos, sendo que os tipos diferentes são utilizados conforme a sua aplicação (quadro 1).

Quadro 1: Tipos de perfis das roscas conforme sua aplicação

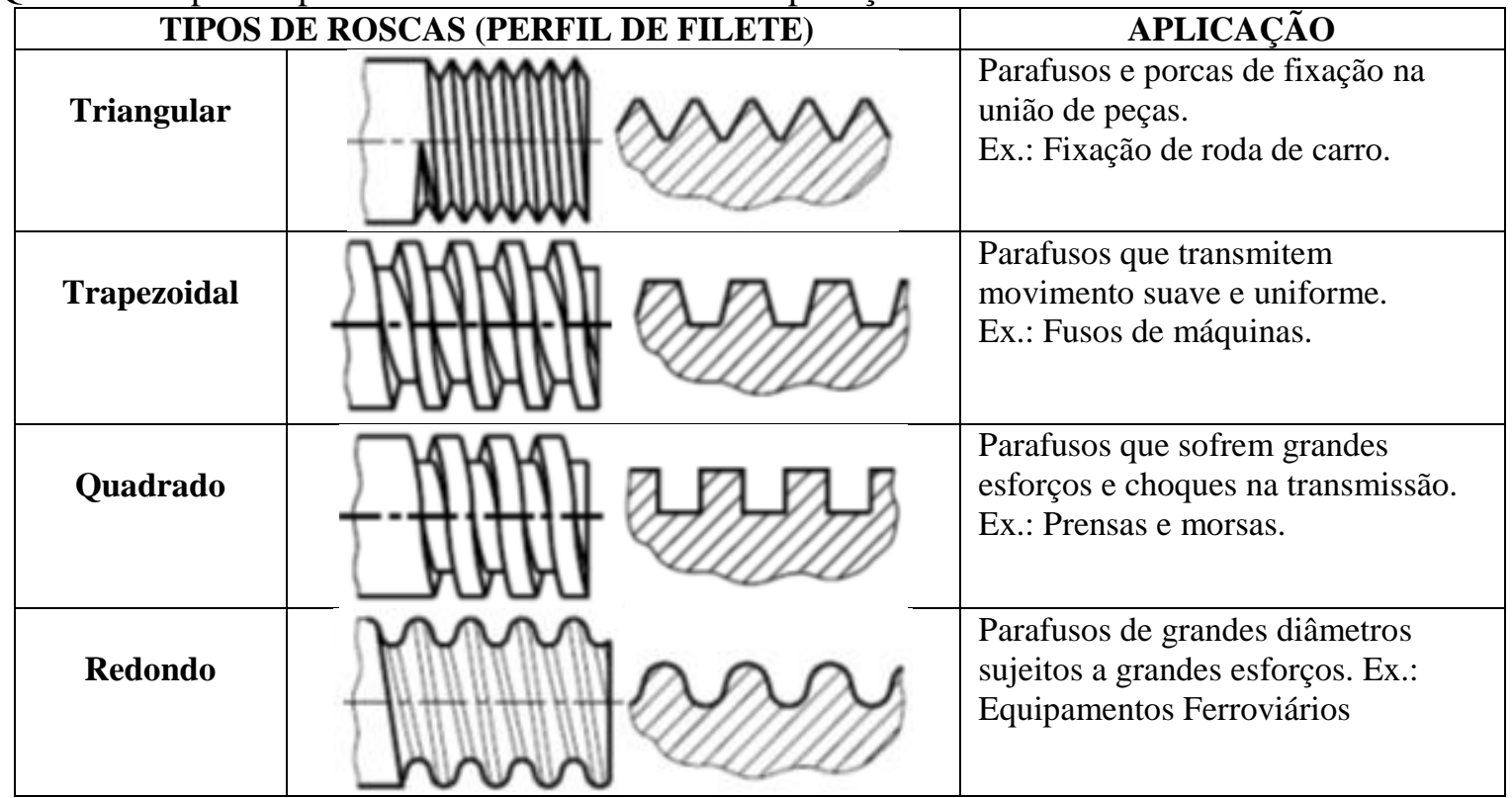




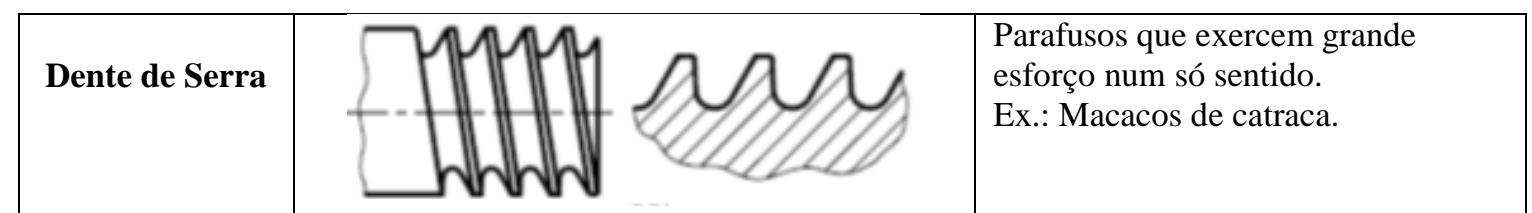

Fonte: Gordo e Ferreira (2012, p.35)

Os parafusos que possuem o objetivo de transmissão de movimento podem ser de dois tipos, parafusos de potência (também chamados de parafusos de avanço ou de fusos) e os parafusos de rosca sem fim (ou simplesmente parafusos sem fim).

Os parafusos de potência são aplicados juntamente com porcas especiais (buchas ou mancais roscados) para simplesmente transformar o movimento angular (rotação) em movimento linear (translação retilínea) de um determinado mecanismo (SHIGLEY; MISCHKE; BUDYNAS, 2005). A figura 1 representa este tipo de parafuso.

Figura 1: Parafusos de avanço com porcas especiais dotadas de flanges

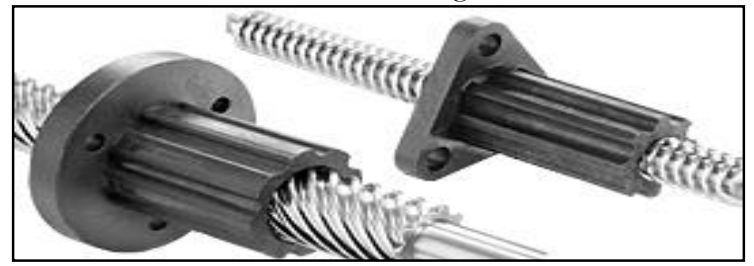

Fonte: Thomson, jul. 2014.

Em qualquer tipo de transmissão é inevitável a perda de potência originada pelos atritos entre as superfícies que estão em contato e também pela agitação do óleo em casos de lubrificação contínua em partes dos elementos de transmissão (MELCONIAN, 2008).

Segundo Norton (2000) alguns experimentos demonstraram que $\mathrm{O}$ coeficiente de atrito entre um parafuso de potência de aço e a bucha de ferro fundido ou bronze vale cerca de 0,15 $\pm 0,05$ (quando lubrificados por óleo).

Shigley, Mischke e Budynas (2005) apresentaram alguns resultados obtidos em trabalhos experimentais com coeficiente de atrito para algumas combinações de materiais para o parafuso e a porca (tabela 1).

Tabela 1: Coeficiente de atrito para combinação do material do parafuso e da porca

\begin{tabular}{ccccc}
\hline Material do parafuso & \multicolumn{4}{c}{ Material da porca } \\
\cline { 2 - 5 } & Aço & Bronze & Latão & Ferro fundido \\
\hline Aço (seco) & $0,15-0,25$ & $0,15-0,23$ & $0,15-0,19$ & $0,15-0,25$ \\
Aço (lubrificado) & $0,11-0,17$ & $0,10-0,16$ & $0,10-0,15$ & $0,11-0,17$ \\
Bronze & $0,08-0,12$ & $0,04-0,06$ & - & $0,06-0,09$ \\
\hline
\end{tabular}

Fonte: Rothbart (1985 apud SHIGLEY; MISCHKE; BUDYNAS, 2005, p.396).

A figura 2 apresenta um gráfico com curvas de eficiência de parafusos de potência com roscas padronizadas Acme padrão americano (trapezoidal) em função do ângulo de avanço do parafuso ( $\lambda$ ) e também em função do coeficiente de atrito.
Analisando o gráfico, percebe-se que quando maior o coeficiente de atrito menor é a eficiência da transmissão. Como os parafusos de potência convencionais são fabricados com ângulos de avanço entre 2 e 5 graus (tabela 2) e que o coeficiente de atrito possui valor próximo a 0,15 , então, pelo gráfico, conclui-se que a eficiência fica em 
torno de $18 \%$ e $36 \%$, valores considerados baixos (NORTON, 2000).

Figura 2: Curvas de Eficiências de parafusos de potência com roscas Acme (Trapezoidal)

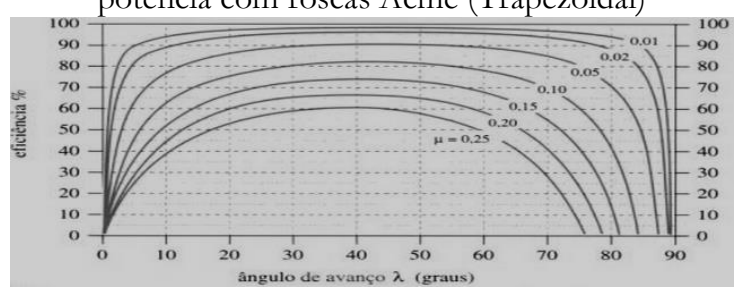

Fonte: Norton (2000, p. 768)
A tabela 2 apresenta alguns valores para o passo de uma rosca Acme padrão americano em função do diâmetro médio do parafuso e do ângulo de avanço $(\lambda)$.

Tabela 2: Diâmetro, passo e ângulo de avanço indicados para parafusos com rosca Acme

\begin{tabular}{ccccccccccc}
\hline Diâmetro (mm) & 6,5 & 8,0 & 9,5 & 12,7 & 15,9 & 19,1 & 22,2 & 25,4 & 31,8 & 38,1 \\
\hline Passo (mm) & 1,6 & 1,8 & 2,1 & 2,5 & 3,2 & 4,2 & 4,2 & 5,1 & 5,1 & 6,4 \\
\hline $\begin{array}{c}\text { Angulo de } \\
\text { avanço (graus) }\end{array}$ & 5,2 & 4,7 & 4,5 & 4,0 & 4,0 & 4,5 & 3,8 & 4,0 & 3,2 & 3,3 \\
\hline
\end{tabular}

Fonte: Shigley, Mischke e Budynas (2005, p.390) e Norton (2000, p.768).

Segundo Norton (2000), para conseguir uma diminuição significativa do atrito e consequentemente uma melhoria considerável da eficiência é empregado o parafuso de esfera (figura 3).

Figura 3: Parafuso de potência com esferas para diminuição do atrito.

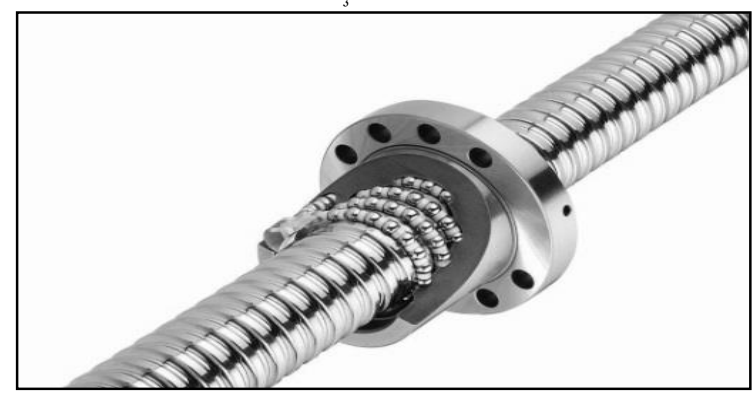

Fonte: MECTROL, 2014

Shigley, Mischke e Budynas (2005) definem que o avanço num parafuso de potência é a distância que a porca se move paralelamente ao eixo do parafuso quando lhe é aplicada uma volta. O parafuso pode possuir uma ou mais entradas e a quantidade de entradas está relacionada com o avanço obtido por volta, isto é, para saber o avanço de um parafuso de potência, basta multiplicar o passo do parafuso (distância entre filetes consecutivos da rosca) pelo número de entradas (figura 4).

Figura 4: representação de uma rosca com quatro entradas

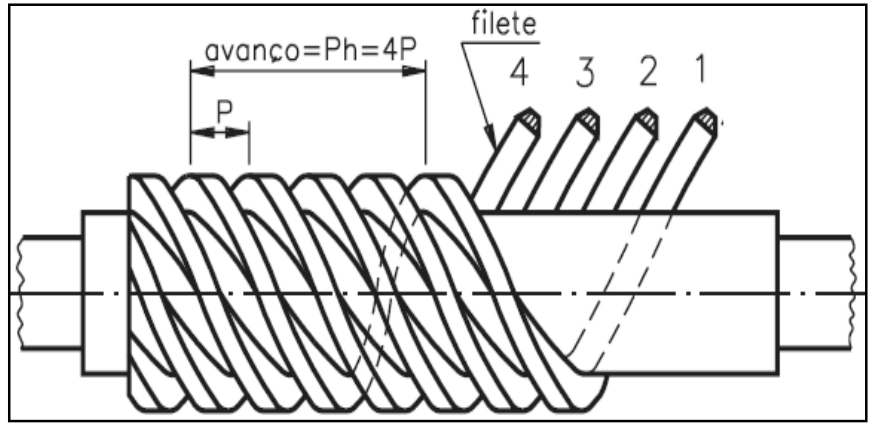

Fonte: Gordo e Ferreira (2012, p.274)

\section{Cálculo da Rotação}

Quando se deseja adquirir um motor para realizar uma atividade cuja transmissão será por parafuso de potência, é necessário estimar a potência, o torque e a rotação de funcionamento que o capacita para realizar determinado trabalho. Para isso necessita-se saber qual o tipo de movimento, qual a massa do corpo que será deslocado, o tipo, o 
diâmetro e o número de entradas adequado do parafuso de potência para a transmissão, a velocidade desejada e até mesmo a aceleração pretendida. O primeiro passo é determinar o tipo de rosca adequado para o parafuso de potência conforme quadro 1 , onde se observa que para transmissão suave deve ser utilizado o de rosca trapezoidal e quando for para transmissão com grandes esforços e/ou choque deve ser utilizado o de rosca quadrada. Depois, estipula-se a quantidade de entradas e o passo adequados para a transmissão em função do avanço (A) desejado. Essa escolha depende da análise da eficiência e do que é encontrado no mercado.

Sabendo o avanço desejado (A) e o espaço linear $(\Delta S)$ que o sistema terá que percorrer, é só determinar a quantidade de voltas $(\mathrm{N})$ que o motor deverá executar para percorrer esse espaço desejado (equação 1).

$$
N=\frac{\Delta S}{A}
$$

Sendo o avanço (A) definido como o produto do passo (p) pelo número de entradas $(\mathrm{Ne})$, então obtém-se a equação 2 :

$$
N=\frac{\Delta S}{p N_{e}}
$$

Com o número de rotações do motor para percorrer o espaço desejado e determinando o tempo que se deseja percorrer esse espaço, pode-se calcular a frequência de rotação do motor utilizando a equação 3 .

$$
f=\frac{N}{\Delta t}
$$

Onde:

$\mathrm{f}=$ frequência;

$\Delta \mathrm{t}=$ intervalo de tempo

Uma vez encontrada a frequência, podese determinar a rotação (n) através da equação 4 (MELCONIAN, 2008, p.18). $n=60 . f$

\section{Cálculo do Torque}

Para a determinação do torque a situação é um pouco mais complicada, pois deve levar em consideração, além do esforço para mover a carga, o esforço para rotacionar o parafuso. Os resultados são diferentes para o movimento vertical ascendente, $\mathrm{O}$ movimento vertical descendente e também para os casos de movimento horizontal. Além disso, devem ser analisadas as características geométricas do parafuso que será utilizado na transmissão.

A figura 5 representa um parafuso de potência com rosca quadrada onde está indicado o diâmetro médio $(\mathrm{dm})$, o passo $(\mathrm{p})$, o ângulo de avanço $(\lambda)$, o ângulo de hélice $(\psi)$, uma porca e a força axial $(F)$ a que o parafuso está submetido.

Figura 5: Parafuso de potência com rosca quadrada

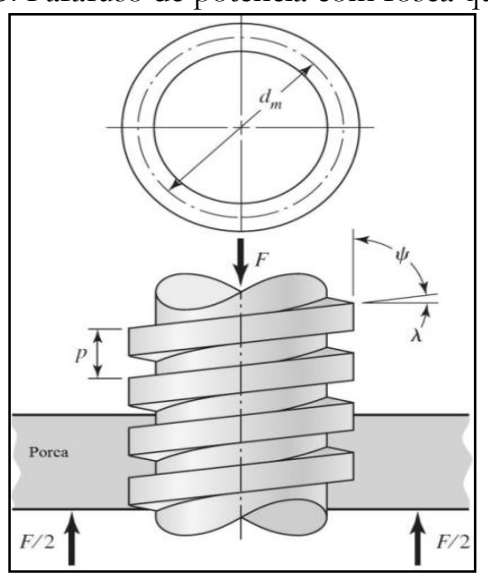

Fonte: Shigley, Mischke e Budynas (2005, p.391)

A figura 6 apresenta um parafuso de potência de rosca trapezoidal (rosca no padrão Acme americano). Observa-se que a força axial $(\mathrm{F})$ agora possui uma componente devido ao ângulo de rosca $(2 \alpha)$. A figura também indica a presença de um colar ou mancal axial que objetiva servir de apoio quando o parafuso é carregado axialmente (SHIGLEY; MISCHKE; BUDYNAS, 2005). 
Figura 6: Parafuso de potência com rosca trapezoidal (rosca no padrão Acme americano)

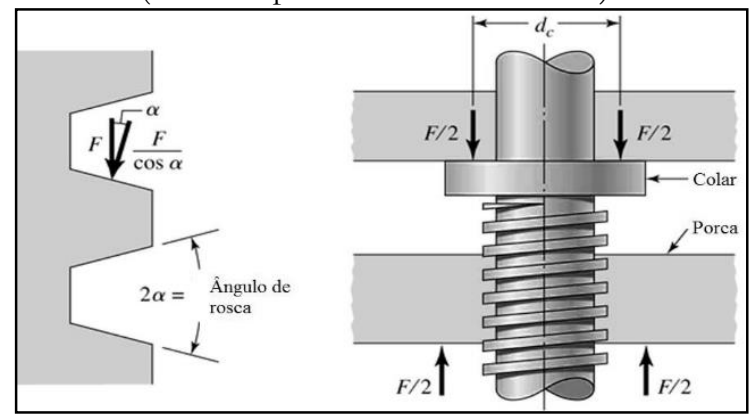

Fonte: Shigley, Mischke e Budynas (2005, p.392)

Segundo Shigley, Mischke e Budynas (2005), para analisar as forças que o parafuso de potência está submetido, basta imaginar um único filete da rosca "desenrolado" (linearizado) por exatamente uma volta, assim, pode-se formar um triângulo retângulo (figura 7) onde a hipotenusa é o comprimento do filete "desenrolado", a base é o comprimento da circunferência do círculo de diâmetro médio de rosca $(\mathrm{dm})$, a altura é o avanço (A) do parafuso e o ângulo formado com a horizontal é o ângulo de avanço $(\lambda)$ no caso do parafuso de rosca quadrada, ou da combinação do ângulo do filete $(\square)$ com o ângulo de avanço ( $\lambda$ ) no caso do parafuso com rosca trapezoidal (Acme) ou outros tipos de roscas, conforme observado nas figuras 5 e 6.

O sistema de forças para um movimento vertical ascendente de um determinado corpo através de um parafuso de potência com rosca quadrada está representado na figura 7 . A figura $7 \mathrm{a}$ apresenta todas as forças envolvidas, a força de atrito (Fat) entre o parafuso e a porca, a força normal $(\mathrm{N})$, a força (Fs) necessária para rotacionar o parafuso e elevar a carga e a força de resistência (R) da carga que será erguida (se o corpo estiver suspenso sem estar guiado, deve-se considerar apenas o peso (W) da carga que será elevada, agora se o corpo estiver guiado deve-se adicionar à força peso a força de atrito correspondente ao movimento na guia). A figura $7 \mathrm{~b}$ apresenta as componentes das forças que estão inclinadas em relação à vertical e/ou em relação à horizontal.

Figura 7: Diagrama de forças que atuam num parafuso de potência com rosca quadrada para um movimento ascendente

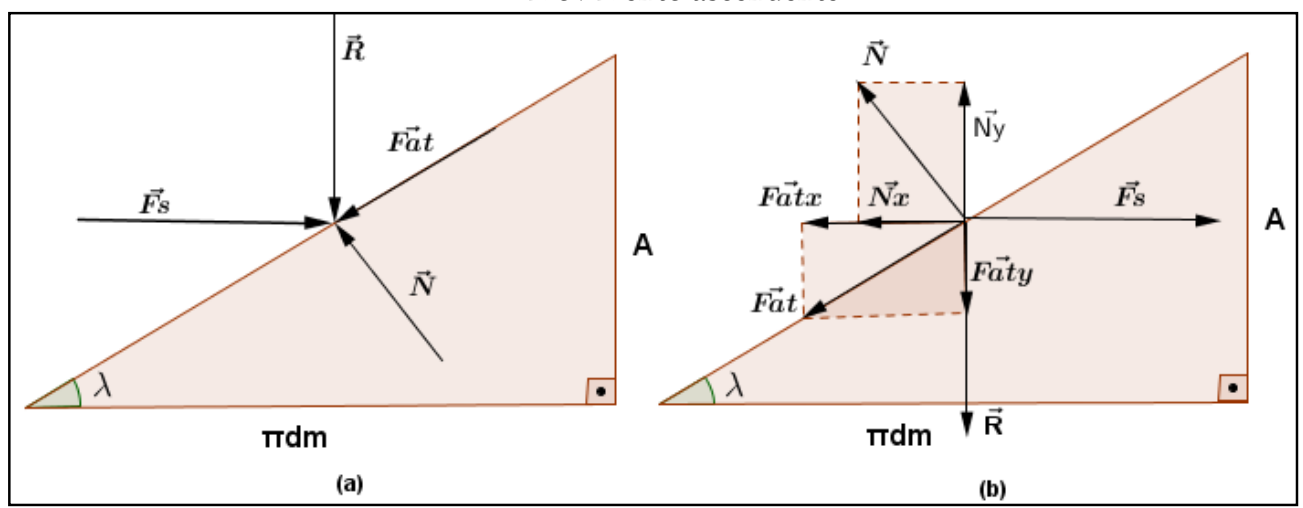

Fonte: Do autor, 2014

Tomando como base a figura $7 \mathrm{~b}$, aplicando a segunda lei de Newton (equação 5) e considerando apenas as forças verticais, então:

$\sum \vec{F}=m \cdot \vec{a}$

$$
N_{y}-F_{a t y}-R=m \cdot a
$$

Separando as componentes em função de seno e cosseno, têm-se:

$$
N \cos \lambda-F_{a t} \sin \lambda-R=m \cdot a
$$


Conforme Hibbeler (2011, p.89), a força de atrito (Fat) é calculada pelo produto do coeficiente de atrito $(\square)$ pela força normal $(\mathrm{N})$, assim:

$$
N \cos \lambda-\mu N \sin \lambda=m \cdot a+R
$$

Isolando a força normal $(\mathrm{N})$ obtém-se a equação 6 .

$$
\begin{aligned}
& N[\cos \lambda-\mu \sin \lambda]=m \cdot a+R \\
& N=\frac{m a+R}{\cos \lambda-\mu \sin \lambda}
\end{aligned}
$$

Fazendo o mesmo procedimento para as forças horizontais da figura $7 b$, têm-se:

$$
\begin{aligned}
\sum \vec{F}= & m \cdot \vec{a} \\
& \quad F_{S}-F_{a t x}-N_{x}=m \cdot a
\end{aligned}
$$

Separando as componentes em função de seno e cosseno, chega-se a equação 7:

$$
F_{S}-F_{a t} \cos \lambda-N \sin \lambda=m \cdot a
$$

Substituindo-se a equação 6 na equação 7 e isolando a força Fs, chega-se a equação 8.

$$
\begin{aligned}
& F_{S} \\
& =m a+\frac{(m a+R) \cdot[\mu \cos \lambda+\sin \lambda]}{\cos \lambda-\mu \sin \lambda}
\end{aligned}
$$

Dividindo-se o numerador e o denominador do membro da direita da equação 8 por $\cos \lambda$ e sabendo que, pelo triângulo retângulo da figura $7, \tan \lambda=\frac{A}{\pi d_{m}}$, então se obtêm a equação 9 que representa a força $(\mathrm{Fs})$ para rotacionar o parafuso e assim elevar uma carga.

$$
\begin{array}{r}
F_{s}=m a+\frac{(m a+R) \cdot\left(\mu+\frac{A}{\pi d_{m}}\right)}{\left(1-\frac{\mu A}{\pi d_{m}}\right)} \\
F_{s}=m a+\frac{(m a+R) \cdot\left(\mu \pi d_{m}+A\right)}{\left(\pi d_{m}-\mu A\right)}
\end{array}
$$

Sendo o torque (MT) definido como o produto da força tangencial pelo raio (MELCONIAN, 2008, p.28) e como nesse caso a força tangencial é a força (Fs) e o raio será a metade do diâmetro médio $(\mathrm{dm})$ do parafuso, então se chega a equação 10 que representa $\mathrm{O}$ torque necessário para rotacionar o parafuso e assim elevar uma carga.

$$
M_{T}=\left[m a+\frac{(m a+R) \cdot\left(\mu \pi d_{m}+A\right)}{\left(\pi d_{m}-\mu A\right)}\right] \cdot \frac{d_{m}}{2}
$$

O sistema de forças para um movimento vertical descendente de um determinado corpo através de um parafuso de potência com rosca quadrada está representado na figura 8. A figura 8a apresenta todas as forças envolvidas, a força de atrito (Fat) entre o parafuso e a porca, a força normal $(\mathrm{N})$, a força (Fd) necessária para rotacionar o parafuso e mover a carga para baixo e a força de resistência (R) da carga que descerá (se o corpo estiver suspenso sem estar guiado, deve-se considerar apenas o peso (W) da carga que descerá, agora se o corpo estiver guiado deve-se subtrair da força peso a força de atrito correspondente ao movimento na guia). A figura 8b apresenta as componentes das forças que estão inclinadas em relação à vertical e/ou em relação à horizontal. 
Figura 8: Diagrama de forças que atuam num parafuso de potência com quadrada para movimento descendente

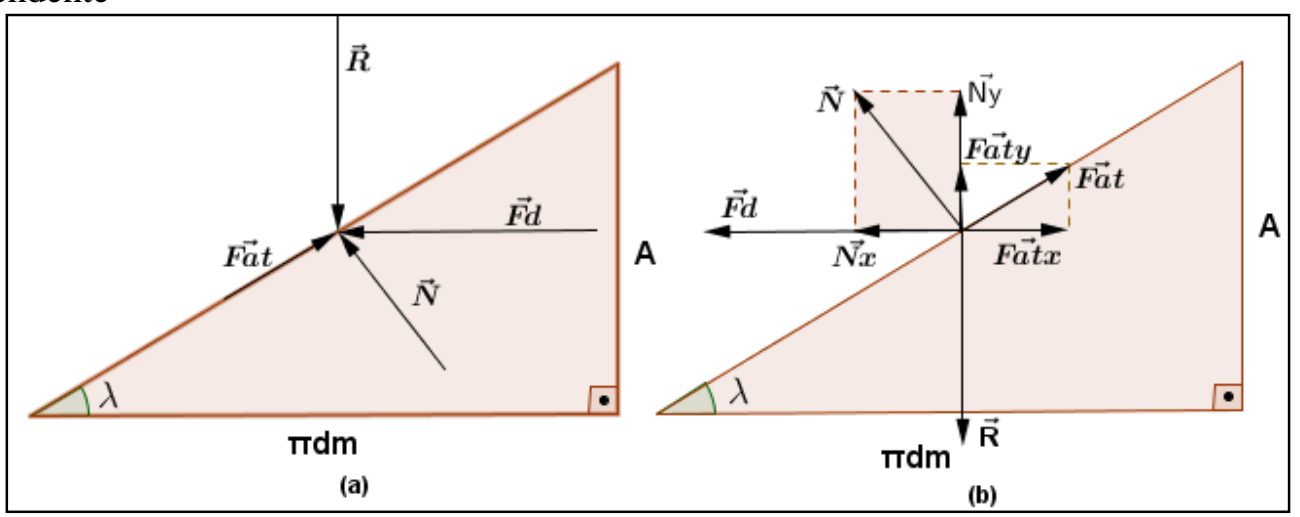

Fonte: Produzido pelo autor, novembro, 2014.

A partir dos vetores apresentados na figura $8 \mathrm{~b}$ pode-se aplicar o mesmo procedimento utilizado no caso do movimento ascendente, chegando-se assim na equação 11 que representa a força $\mathrm{Fd}$ e na equação 12 que representa o torque (MT) necessário para rotacionar o parafuso e fazer com que a carga desça.

$$
\begin{gathered}
F_{d}=m a+\frac{(m a+R) \cdot\left(\mu \pi d_{m}-A\right)}{\left(\pi d_{m}+\mu A\right)} \\
M_{T}=\left[m a+\frac{(m a+R) \cdot\left(\mu \pi d_{m}-A\right)}{\left(\pi d_{m}+\mu A\right)}\right] \cdot \frac{d_{m}}{2}
\end{gathered}
$$

O sistema de forças para um movimento horizontal de um determinado corpo através de um parafuso de potência com rosca quadrada, que também deverá estar na horizontal, está representado na figura 9. A figura 9a apresenta todas as forças envolvidas, a força de atrito (Fat) entre o parafuso e a porca, a força normal $(\mathrm{N})$, a força (Fh) necessária para rotacionar o parafuso e mover uma carga horizontalmente e a força de atrito (Fatc) da carga que será movida em relação à superfície em que estará apoiada e/ou até mesmo guiada. A figura $9 \mathrm{~b}$ apresenta as componentes das forças que estão inclinadas em relação à vertical e/ou em relação à horizontal.

Figura 9: Diagrama de forças que atuam num parafuso de potência com rosca quadrada para movimento horizontal

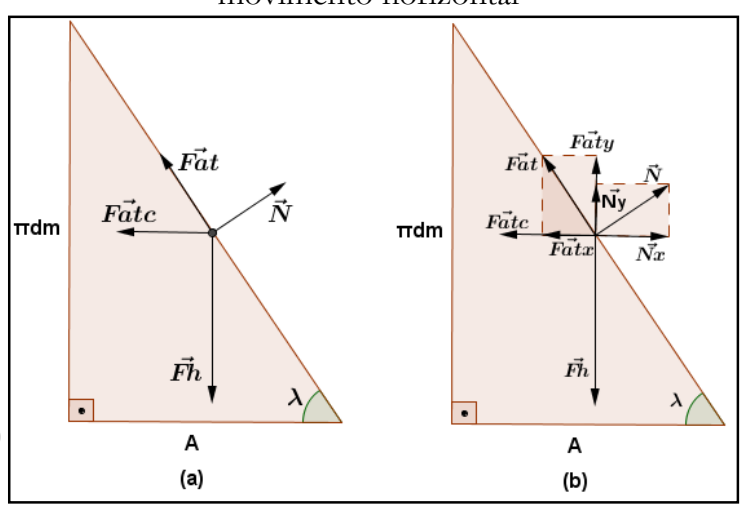

Fonte: Elaborado pelo autor

Tomando como base a figura 9b, aplicando a segunda lei de Newton e considerando apenas as forças verticais, então:

$$
\begin{aligned}
& \sum \vec{F}=m \cdot \vec{a} \\
& \quad F_{\mathrm{h}}-N_{y}-F_{a t y}=m \cdot a
\end{aligned}
$$

Separando as componentes em função de seno e cosseno, têm-se:

$$
F_{\mathrm{h}}-N \sin \lambda-\mu N \cos \lambda=m \cdot a
$$

Isolando a força normal $(\mathrm{N})$ obtém-se a equação 13. 


$$
\begin{aligned}
& F_{\mathrm{h}}-m \cdot a=N[\sin \lambda+\mu \cos \lambda] \\
& N=\frac{F_{\mathrm{h}}-m \cdot a}{\sin \lambda+\mu \cos \lambda}
\end{aligned}
$$

Fazendo o mesmo procedimento para as forças horizontais da figura 9b, têm-se:

$$
N_{x}-F_{a t x}-F_{a t c}=m \cdot a
$$

Separando as componentes em função de seno e cosseno, chega-se a equação 14 :

$N \cos \lambda-\mu N \sin \lambda-\mu_{c} N_{c}=m \cdot a$

Onde é o coeficiente de atrito entre o corpo que se deslocará e a superfície de apoio, é o coeficiente de atrito entre o parafuso e a porca e a força normal referente ao corpo que se deslocará horizontalmente, sendo que essa força normal é igual à força peso dada por .

Substituindo-se a equação 13 na equação 14, isolando a força $F h$ e rearranjado conforme as deduções anteriores obtêm-se a equação 15 que representa a força $(\mathrm{Fh})$ para rotacionar o parafuso e assim deslocar a carga horizontalmente.

$F_{h}=m a+\frac{m\left(a+\mu_{c} g\right) \cdot\left(\mu \pi d_{m}+A\right)}{\pi d_{m}-\mu A}$

E, consequentemente, chega-se à equação 16 que representa o torque necessário para rotacionar o parafuso e assim deslocar a carga horizontalmente.

$M_{T}=\left[m a+\frac{m\left(a+\mu_{c} g\right) \cdot\left(\mu \pi d_{m}+A\right)}{\pi d_{m}-\mu A}\right] \cdot \frac{d_{m}}{2}$

As equações 10, 12 e 16 representam o cálculo do torque para parafusos com roscas quadradas onde as forças normais são paralelas ao eixo do parafuso. No caso de roscas Acme (figura 6) ou outros tipos de roscas, a força de resistência fica inclinada em relação ao eixo do parafuso devido ao ângulo de rosca $(2 \alpha)$ e ao ângulo de avanço $(\lambda)$. Como o ângulo de avanço é muito menor que o ângulo da rosca, então ele pode ser desprezado e o efeito desse ângulo é de aumentar a força de resistência devido à ação de calço da rosca, pois aumenta a força normal nos filetes (figura 6) e consequentemente a força de atrito (SHIGLEY; MISCHKE; BUDYNAS, 2005). Fazendo uma dedução similar às do parafuso com rosca quadrada, chegam-se as equações 17, 18 e 19 que representam os torques aproximados (porque está sendo considerado desprezível o ângulo de avanço) no movimento vertical ascendente, descendente e horizontal respectivamente, para um parafuso de rosca Acme padrão americano.

Para parafusos de potência, a rosca Acme não é tão eficiente quanto à quadrada, devido à fricção adicional decorrente da ação de calço (ou cunha), mas frequentemente é preferida por ser mais fácil de usinar e por permitir o uso de uma porca de partida, que pode ser ajustada para compensar o desgaste (SHIGLEY; MISCHKE; BUDYNAS, 2005, p.392).

$$
M_{T}=\left[m a+\frac{(m a+R) \cdot\left(\mu \pi d_{m} \sec \alpha+A\right)}{\left(\pi d_{m}-\mu A \sec \alpha\right)}\right] \cdot \frac{d_{m}}{2}
$$

$$
M_{T}=\left[m a+\frac{(m a+R) \cdot\left(\mu \pi d_{m} \sec \alpha-A\right)}{\left(\pi d_{m}+\mu A \sec \alpha\right)}\right] \cdot \frac{d_{m}}{2}
$$

$$
M_{T}=\left[m a+\frac{m\left(a+\mu_{c} g\right) \cdot\left(\mu \pi d_{m} \sec \alpha+A\right)}{\pi d_{m}-\mu A \sec \alpha}\right] \cdot \frac{d_{m}}{2}
$$

Observe que para $\square=0$ (no caso de roscas quadradas) as equações 17,18 e 19 tornam-se as equações 10, 12 e 16 respectivamente, isto é, as equações 17, 18 e 19 são as equações gerais para os torques em parafusos de potência, sendo que os termos que estão entre colchetes representam a força para rotacionar o parafuso. Vale destacar que 
o parafuso com roscas Acme o ângulo de rosca $(2 \alpha)$ possui um valor padrão de $29^{\circ}$ (SHIGLEY; MISCHKE; BUDYNAS, 2005).

Quando um parafuso é dotado de um mancal axial ou colar para apoio devido a esforços axiais (figura 6), deve ser considerado um torque adicional dado pela equação 20, não importando o tipo de rosca nem o tipo de movimento do parafuso (NORTON, 2000, p.766).

$$
M_{T}=\frac{R \mu_{\text {colar }} d_{c}}{2}
$$

\section{Onde:}

$\mathrm{R}=$ força de resistência que age no sentido contrário do movimento (peso, atrito...);

$\mu_{\text {colar }}=$ coeficiente de atrito entre o colar e a superfície de apoio;

dc $=$ diâmetro do colar médio (figura 6).

\section{Cálculo da Potência}

Conforme Resnick, Halliday e Krane (2003, p.264), a potência mecânica (P) é definida como o produto da força tangencial pela velocidade (equação 21), então:

$P=F_{T} \cdot v$

Segundo Melconian (2008, p.31) o torque é diretamente proporcional à potência e inversamente proporcional à rotação (equação 22).

$$
M_{T}=\frac{30 P}{\pi n}
$$

Isolando-se a potência encontra-se outra maneira de calcular a potência, agora em função do torque e da rotação (equação 23).

$$
P=\frac{M_{T} \pi n}{30}
$$

Essa potência mecânica (P) calculada pela equação 21 ou pela equação 23 é a potência necessária para realizar o trabalho mecânico, isto é, é a potência útil. A potência de saída (PS) no eixo do motor deve ser determinada considerando a eficiência $(\varepsilon)$ da transmissão por parafusos de potência. A equação 24 representa o cálculo da potência de saída.

$$
P_{S}=\frac{P}{\varepsilon}
$$

O torque que o motor deverá possuir para realizar essa atividade é calculado através da equação 22, mas já considerando a potência de saída (PS) no eixo do motor.

\section{Cálculo da Eficiência}

A eficiência ou rendimento de um parafuso de potência é a razão entre o trabalho de saída e o trabalho de entrada (NORTON, 2000, p.767). O trabalho de entrada ( $\tau$ e) pode ser definido como o produto do torque pelo deslocamento angular $(\Delta \varphi)$ em radianos (equação 25 ).

$$
\tau_{e}=M_{T} \Delta \varphi
$$

Já o trabalho de saída ( $\tau s)$ pode ser definido como o produto da força de resistência $(\mathrm{R})$ pelo deslocamento linear $(\Delta \mathrm{S})$, conforme equação 26.

$$
\tau_{s}=R \Delta S
$$

Assim, a eficiência de um parafuso de potência é dada pela equação 27 . 


$$
\varepsilon=\frac{R \Delta S}{M_{T} \Delta \varphi}
$$

Como em uma volta completa o deslocamento angular equivale a $2 \square$ e o deslocamento linear equivale ao avanço linear (A), basta substituir a equação 27 por esses parâmetros e têm-se outra forma de representar a eficiência (equação 28).

$$
\varepsilon=\frac{R A}{M_{T} 2 \pi}
$$

Substituindo a equação 17 na equação 28, desconsiderando a aceleração linear e sabendo que , assim, encontra-se a equação 29 que é a equação geral para estimar a eficiência da transmissão por parafuso de potência (NORTON, 2000, p.768).

$$
\varepsilon=\frac{\cos \alpha-\mu \tan \lambda}{\cos \alpha+\mu \cot \lambda}
$$

\section{Considerações finais}

Neste artigo foi demonstrado como deve ser o procedimento para calcular a rotação, o torque e a potência que um motor deverá possuir para executar determinada atividade utilizando parafuso de potência ou de avanço como elemento de transmissão, através da indicação ordenada de todos os cálculos envolvidos para tal dimensionamento, situação não encontrada na literatura. Além disso, foi também ressaltado como se calcula a eficiência mecânica alcançada pelo parafuso de potência.

As equações encontradas para o torque estão mais completas e precisas com relação às equações disponíveis nos livros de projetos mecânicos, pois além de ter a inclusão da aceleração para o motor partir do repouso e atingir a rotação máxima, encontra-se também o torque referente ao movimento horizontal.

Vale destacar que o artigo abordou o dimensionamento de algumas características do parafuso de potência e do motor, não sendo apresentado o dimensionamento de todas as características geométricas desse parafuso, pois isso já é encontrado com muita clareza em vários livros, e nem da escolha do tipo de motor, pois não foi $\mathrm{o}$ foco do trabalho.

\section{Bibliografia}

1. DEL TORO, Vicent. Fundamentos de Máquinas Elétricas. Rio de Janeiro: LTC, 2009.

2. GORDO, Nívia; FERREIRA, Joel. Mecânica: Elementos de Máquinas. São Paulo, 2012, 414p. Apostila do Telecurso 2000 - FIESP, CIESP, SESI, SENAI, Irs.

3. HALLIDAY, David; RESNICK, Robert; WALKER, Jearl. Fundamentos de Física Eletromagnetismo. 6. ed. Rio de Janeiro: LTC, 2003.

4. HIBBELER, Russell C. Dinâmica: Mecânica para Engenharia. 12 ed. São Paulo: Pearson Prentice Hall, 2011.

5. MECTROL - Produtos - Disponível em <http://www.mectrol.com.br/mectrol/pt/ produto/listar/ categoria/1/fusos-deesferas.html> acesso em jul. 2014.

6. MELCONIAN, Sarkis. Elementos de máquinas. 9. ed. São Paulo: Érica, 2008. 
NILSSON, James W; RIEDEL, Susan A.

Circuitos Elétricos. 8. ed. São Paulo: Pearson

Prentice Hall, 2008.

7. NORTON, Robert L. Projeto de Máquinas

- Uma Abordagem Integrada. 2. ed. São Paulo: Artmed, 2000.

8. RESNICK, Robert; HALLIDAY, David;

9. KRANE, Kenneth S. Física 1. 5. ed. Rio de Janeiro: LTC, 2003.

10. ROTHBART, H. A.. Mechanical Design and Systems Handbook. 2nd ed. New York: McGraw-Hill, 1985.
11. SHIGLEY, Joseph E.; MISCHKE, Charles R.; BUDYNAS, Richard G. Projeto de Engenharia Mecânica. 7. ed. Porto Alegre: Bookman, 2005.

12. THOMSON - MTS Flange Mount Supernuts - Disponível em <http://www. thomsonlinear.com/website/com/eng/prod ucts/ball_screws_and_lead_screws/lead_scr ews/mts.php > acesso em jul. 2014.

13. VAZ, Frederico S. O. Máquinas Elétricas. Florianópolis: SENAI, 2010. 